\title{
Féeries
}

Études sur le conte merveilleux, XVII $-\mathrm{XIX}{ }^{\mathrm{e}}$ siècle

$10 \mid 2013$

Conte et croyance

\section{La vérité cabalistique : dynamique des croyances et puissance des faitiches dans Lamekis de Mouhy}

Cabalistic Truth: The Dynamics of Beliefs and the Power of Factishes in Mouhy's Lamekis

Yves Citton

\section{OpenEdition}

\section{Journals}

Édition électronique

URL : http://journals.openedition.org/feeries/899

ISSN : 1957-7753

Éditeur

UGA Éditions/Université Grenoble Alpes

Édition imprimée

Date de publication : 20 septembre 2013

Pagination : 195-214

ISBN : 978-2-84310-253-0

ISSN : $1766-2842$

Référence électronique

Yves Citton, «La vérité cabalistique : dynamique des croyances et puissance des faitiches dans Lamekis de Mouhy », Féeries [En ligne], 10 | 2013, mis en ligne le 20 mars 2015, consulté le 07 septembre 2020. URL : http://journals.openedition.org/feeries/899

(c) Féeries 
Yves Citton

Université Grenoble Alpes, UMR LiRE

\section{LA VÉRITÉ CABALISTIQUE : DYNAMIQUE DES CROYANCES ET PUISSANCE DES FAITICHES DANS LAMEKIS DE MOUHY}

S 'IL EST UN TEXTE QUI PEINE à susciter la croyance chez un lecteur du $\mathrm{XxI}^{\mathrm{e}}$ siècle, c'est sans doute le roman Lamekis que Charles de Fieux, chevalier de Mouhy (I70I-I784), publie en huit parties entre I735 et I738. Mathieu Brunet n'a pas tort d'inscrire ce roman peuplé de contes — ou cet interminable conte à la taille de roman-fleuve — au registre des "monstres littéraires" et autres "textes illisibles» produits par un XVIII ${ }^{\mathrm{e}}$ siècle bien moins rationaliste qu'on ne le dit $^{\mathrm{I}}$. Son sous-titre promettant Les Voyages extraordinaires d'un Égyptien dans la terre intérieure avec la découverte de l'île des Sylphides nous laisse bien attendre du surnaturel et du prodige, au sein d'un merveilleux inscrit sous les auspices des esprits aériens du Comte de Gabalis, mais cela ne suffit nullement à nous prévenir de l'erratisme générique, thématique et énonciatif qui caractérise ce récit hors-norme. Même s'il est difficile de reconstituer la façon dont un tel texte pouvait être lu dans le deuxième tiers du XVIII ${ }^{\mathrm{e}}$ siècle, dans un contexte où la romancie était pétrie de merveilleux, l'extravagance narrative de ce récit nous pose aujourd'hui des problèmes intéressants sur le statut de la croyance, telle qu'elle peut être à la fois mise en scène et mobilisée, dans l'espace fictionnel.

I. M. Brunet, L'Appel du monstrueux. Pensées et poétiques du désordre en France au XVIII siècle, Louvain, Peeters, "La République des Lettres 32", 2008, p. II3-I2O. Pour de bonnes analyses synthétiques de Lamekis, voir Y. Giraud, "Monstres et merveilles au centre de la terre : les fantastiques aventures du Chevalier de Mouhy", dans Studi di letteratura francese, n 13 (1987) et P. Fitting, "Imagination, Textual Play and the Fantastic in Mouhy's Lamekis", Eighteenth-Century Fiction, $\mathrm{n}^{\circ}$ 5-4 (1993), p. 3II-329. Un bel ouvrage collectif faisant le point sur nos connaissances actuelles de Mouhy est paru récemment sous la direction de J. Herman et al., Le Chevalier de Mouhy. Bagarre et bigarrure, Amsterdam, Rodopi, 20Io, comprenant trois études consacrées à Lamekis. E. Sempère met Lamekis (ainsi que Gongam de Laurent Bordelon) au cœur du dernier chapitre, consacré à "La crédulité et le désordre de la fiction", de son ouvrage De la merveille à l'inquiétude : le registre du fantastique dans la fiction narrative $d u$ XVIII ${ }^{e}$ siècle, Presses universitaires de Bordeaux, 2009. 


\section{Un défi à toute croyance}

Suivant qu'on s'attache davantage à l'intrigue amoureuse ou à l'aventure intellectuelle, on résumera Lamekis en y voyant soit un mari qui finit par retrouver la parfaite épouse qu'il avait accusée à tort de l'avoir trompé, soit un renoncement (pleinement assumé) à acquérir la clarté d'esprit et l'immortalité promises par une certaine conception de la philosophie.

La ligne principale du récit suit l'Égyptien Lamekis dans un voyage qu'il entreprend avec son compagnon Sinoüis afin de retrouver son épouse Clemelis, qu'il avait poignardée en croyant à tort qu'elle le trompait avec Motacoa, roi des Abdales. À la suite de la traditionnelle tempête initiale, le navire qui les emmène d'Égypte au royaume des Abdales (où vit Clemelis) se voit toutefois soudainement emporté par une colonne d'eau (un tsunami) qui le fait échouer au sommet d'un arbre dans l'île des Sylphides. Les interactions qu'entretiennent les deux voyageurs avec les Sylphes qui peuplent l'île leur font comprendre qu'ils subissent une épreuve capable de leur assurer l'immortalité, pour autant qu'ils sachent réprimer leurs désirs sensuels et ne pas reculer devant la perspective de la douleur physique. Un philosophe du nom de Dehahal leur sert d'initiateur et de guide au sein de cette épreuve. Il leur raconte comment il est lui-même parvenu, seul parmi tous les humains, à acquérir l'immortalité en s'exposant à une série de supplices culminant en une scène où il s'est vu écorché vif, la peau arrachée, le corps morcelé. Alors que le faible Sinoüis succombe très tôt aux tentations de la chair que lui présentent des sylphes noirs qui le séduisent avec force festins et créatures enchanteresses, Lamekis tient bon à travers toutes les manipulations de son désir. En revanche, au moment où Dehahal le juge prêt pour la dernière grande épreuve de l'écorchage à vif, le protagoniste recule devant la perspective de cette souffrance surhumaine, que lui avait dépeinte le philosophe initiateur. Celui-ci le maudit pour sa faiblesse, avant de le condamner à se métamorphoser en serpent et à «ramper jusqu’à ce qu'une femme fidèle lui rende sa première forme ${ }^{2}$ ».

Lamekis-serpent retrouve alors son compagnon Sinoüis (transformé luimême en hibou), tous deux parviennent à rejoindre l'innocente Clemelis à la cour de Motacoa, et après quelques derniers retournements, ils reprennent leur forme humaine. Lamekis parvient à dévoiler les ruses du fourbe Zelimon, dont les mensonges avaient suscité son injuste jalousie envers

2. C. de Fieux, chevalier de Mouhy, Lamekis, ou Les Voyages extraordinaires d'un Égyptien dans la terre intérieure avec la découverte de l'îsle des Sylphides, Paris, puis La Haye, I735-1738, 8 volumes, volume $\mathrm{V}$, page ior (noté désormais $\mathrm{V}$, IOI). 
sa fidèle épouse, il rentre dans les faveurs du roi, obtient la punition de Zelimon (transformé à son tour en hibou) et finira sa vie en "bon gouvernant». Le philosophe Dehahal réapparaît toutefois pour préciser qu'il n'accordera pas l'immortalité à Lamekis parce que celui-ci n'a pas su résister à son désir de vengeance et n'a pas demandé au roi la grâce de Zelimon, alors qu' "il y a plus de gloire à pardonner qu'à punir" (VIII, I56). Ici aussi, toutefois, loin de regretter un choix qui l'empêche de s'élever au statut supérieur du philosophe, Lamekis paraît plutôt se féliciter de son choix : «malgré l'avis de Dehahal, je persistai dans mon ressentiment, je m’en réjouis dans le secret de mon cœur, et je décidai que si le Ciel me laissait maître du sort de ce traître, il ne reprendrait jamais sa première forme ${ }^{3}$ » (VIII, I57).

Au sein de ce premier niveau narratif, Lamekis apparaît donc comme un personnage qui résiste obstinément au devenir-esprit-philosophe que faisait miroiter le récit à travers les épisodes situés dans l'île des Sylphides. Cette obstination rejoint l'invraisemblable entêtement dont il fait preuve dans sa jalousie envers une épouse que tous les indices tendaient à innocenter. Une telle constance dans la jalousie ou dans la soif de vengeance est d'autant plus frappante qu'elle intervient dans un univers où tout se voit chamboulé à chaque instant par les événements les plus imprévisibles et les plus cataclysmiques. Le roman de Mouhy s'inscrit encore dans une époque où le romanesque est synonyme d'invraisemblances choquantes, d'outrances stylistiques et de renversements constants, qui nous lassent vite que parce que leur radicale imprédictibilité nous apparaît elle-même comme conventionnelle et attendue.

Autour de cette intrigue relativement simple — Lamekis retrouve son épouse après un détour par l'île des Sylphides qui l'a transformé en serpent - se greffent en effet de (trop) nombreux récits annexes et enchâssés retraçant les années antérieures de la vie du protagoniste ainsi que l'histoire du roi Motacoa et de son épouse, Nasilaé. Le roman dans son ensemble a l'allure d'une tempête sans fin, au sein de laquelle les personnages et le lecteur se voient ballottés d'un monde à l'autre, d'une période à l'autre, de catastrophes en catastrophes, de tromperies en traitrises, et d'illusions en hallucinations. Dans les mœurs bizarres des peuples visités, dans les multiples monstres rencontrés en chemin, dans la soudaineté des retournements

3. Sur les enjeux de cette initiation refusée, je renvoie à Y. Citton, «Inspiration et renoncement dans Lamekis", dans J. Herman et al., Le Chevalier de Mouhy. Bagarre et bigarrure, ouvr. cité, p. $153-167$. 
de situation, tout est fait pour exacerber des effets de surprise et d'étonnement, dont l'effet se tarit toutefois par leur multiplication même.

On voit ainsi le père de Lamekis se faire persécuter par la reine Sémiramis, dans une guerre qui met aux prises un pouvoir religieux caché dans des souterrains et un pouvoir politique l'attaquant depuis la surface; on voit le roi Motacoa devoir affronter, également dans les souterrains de la "terre intérieure» annoncée par le titre, un royaume d'hommes-vers, puis d'hommescrapauds, qu'il vainc grâce à son fidèle chien Falbao; on voit Lamekis lui-même se faire recueillir dans un nid d'aigles géants, par lequel il se fait adopter et dont il parvient à dompter l'aiglon devenu orphelin, aiglon qu'il chevauche pour aller venger la mort de son père en abattant le pouvoir de Sémiramis.

L'impression générale qu'en retire le lecteur (moderne) est celle d'être immergé dans un univers chaotique, parfaitement imprévisible, sur lequel on n'a donc aucune maîtrise et que l'on subit sur le mode du cauchemar. Conformément au dispositif méta-fictionnel brillamment analysé par Jean-Paul Sermain ${ }^{4}$, le problème de la croyance à cet univers parfaitement invraisemblable est théorisé par le roman lui-même. Les protagonistes se demandent souvent s'ils sont en train de rêver ou s'ils sont éveillés, le récit évoluant selon des alternances simples entre horreurs et évanouissements, espoirs trompeurs et cruelles déconvenues, révoltes et résignations, selon une dynamique qu'Emmanuelle Sempère a judicieusement située à la charnière du merveilleux et du fantastique, déjouant toute frontière stable entre ce qui relève de la conviction, de l'empathie, de la crédulité et de l'incrédulité. La complaisance avec laquelle Mouhy représente, détaille et répète des scènes d'humiliation et de supplice contribue sans doute grandement à cette impression d'inconfort et de malaise; le choix des noms propres bizarroïdes et radicalement non-intuitifs qu'il attribue à ses personnages (et dont il modifie souvent la graphie d'un volume à l'autre du roman) achève de plonger le lecteur dans le désarroi que cause la perte de tout repères. Comment croire à quoi que ce soit, dès lors que tout est

4. J.-P. Sermain, Métafictions (I670-I730). La réflexivité dans la littérature d'imagination, Paris, Champion, 2002.

5. En rapprochant l'écriture de Mouhy du procédé de l'anamorphose (productrice de distorsions, de discordances, d'équivoques et de non-sens), Emmanuelle Sempère fait de la déstabilisation des croyances et des attentes du lecteur l'enjeu central de Lamekis : «Le roman semble jouer à résister à l'entreprise du lecteur, de même qu'il semble mettre en scène la résistance à l'écriture de la fiction : plus que l'interrogation fantastique sur l'être et le réel, c'est le geste même de l'invention fictionnelle qui est au cœur du travail de Mouhy." (De la merveille à l'inquiétude, ouvr. cité, p. 531, ainsi que $436,509$ et 513.$)$ 
fait pour saper les certitudes à partir desquelles nous essayons de séparer la vérité de l'illusion? C'est l'une des questions que met en scène ce roman aussi fascinant que déroutant.

\section{Puissance des apparences}

Je laisserai de côté l'aspect à mes yeux le plus intéressant de ce roman — la mise en scène des "intelligences» sous la figure de Sylphes ${ }^{6}$ — pour me concentrer ici sur la façon dont, à travers ses différents niveaux narratifs, le texte nous invite à problématiser la croyance d'une façon qui résonne intimement avec ce que peuvent (re)découvrir nos sciences sociales contemporaines. Sans avoir assez de temps pour mettre en place les subtilités du cadre énonciatif (éminemment réflexif) déployé par Mouhy ${ }^{7}$, je vais sélectionner quelques épisodes et citations qui présentent à mes yeux l'intérêt majeur de reconfigurer les paramètres à l'aide desquels nous jugeons les phénomènes de croyance (d'illusion, de naïveté, d'apparence, de vérité, etc.).

Je commencerai par un passage où le philosophe Dehahal déclare à Lamekis vouloir "rapporter [s] on histoire afin qu' elle serve d'exemple», ce qui suscite une note de l'auteur qui noue intimement les ordres narratifs, moraux et politiques :

Dehahal donne une grande leçon dans ce Passage à ceux que le ministère charge de la conduite des autres hommes, en leur faisant connoître que le bon exemple est le plus fort de tous les moyens dont on peut se servir pour les corriger \& pour les amener à la perfection. (IV, 88)

On retrouve ici des traces du paradigme de l'exemplarité qui a joué un rôle si important dans la littérature de la Renaissance ${ }^{8}$ (et qui se voit, bien entendu, surimposé ici de plusieurs couches d'ironie et d'auto-parodie). La puissance de moralisation contenue dans un récit dépendra de la perception de son

6. Sur cette scénographie sylphique, voir, entre autres, M. Delon, "Introduction» à Sylphes et sylphides, Paris, Desjonquères, 1999; I. Mattazzi, La magia come maschera di Eros. Silfidi, demoni e seduttori nella Francia del Settecento, Bergamo, Sestante Edizioni, 2007; et Y. Citton, "Spirits across the Channel. The Staging of Collective Mental Forces in Gabalistic Novels from Margaret Cavendish to Charles Tiphaigne de la Roche", dans Comparatio. Zeitschrift für Vergleichende Literaturwissenschaft, $\mathrm{n}^{\circ}$ I:2 (2009), Heidelberg Universitätsverlag, p. 29I-319.

7. Voir sur cette question la belle analyse de M. Bokobza Kahan, «Intrusions d'auteur et ingérences de personnages : la métalepse dans les romans de Bordelon et de Mouhy", dans Eighteenth Century Fiction, Volume i6-4 (2004), p. 648-654.

8. Voir sur ce point le bel ouvrage de T. Hampton, Writing from History. The Rhetoric of Exemplarity in Renaissance Literature, Ithaca, Cornell University Press, 1990. 
exemplarité, laquelle ne sera pas directement liée à l'existence historique des personnages mis en scène. De même qu'une fable animale peut véhiculer une morale édifiante indépendamment de son irréalisme de surface, de même un récit fictionnel peut-il contribuer à «corriger» les hommes et à "les amener à la perfection». En d'autres termes : on peut ne pas croire à la réalité d'une fable, et néanmoins croire à la leçon morale que son intrigue illustre.

Cette "grande leçon" de politique ne se contente pas de renverser les rapports de pouvoir entre le Prince et le Poète. Elle met surtout en lumière tout ce que le pouvoir politique doit à la sphère du spectacle qui, au fil des récits qu'elle met en circulation, fraie les sensibilités morales des citoyens, oriente leurs aspirations, stimule sélectivement leurs indignations et sculpte leurs espoirs. Les fables que nous lisons configurent nos repérages existentiels en termes de justice et d'injustice à chaque fois qu'elles tracent et retracent un cheminement narratif entre les difficultés de la vertu et les écueils du vice.

Une fiction perçue comme exemplaire par un certain public fonctionne comme un attracteur de réalité : indépendamment de son irréalité originelle, elle pourra faire advenir dans la réalité des types de comportements qui en étaient auparavant exclus. S’il faut un pouvoir institutionnel (d'ordre politique) pour inscrire un sujet dans un réseau de communication où il puisse être reconnu comme tel, il faut également des récits (toujours plus ou moins fictifs) pour permettre aux institutions sociopolitiques de prendre forme et réalité au sein d'une multitude. Il n'y a pas ici création ex nibilo, mais un processus de concrescence (susceptible parfois d'accélérations dramatiques) qui accumule, approfondit, surimpose, redirige des frayages successifs qui en arrivent progressivement à pouvoir canaliser les sensibilités et les affects.

Faire d'un tel processus de concrescence le principe même du pouvoir constituant par lequel s'expliquent l'émergence et l'évolution de nos institutions sociales, et considérer l'exemplarité narrative comme le grain de sable autour duquel prennent forme et réalité les (très imparfaites) perles institutionnelles qui organisent nos sociétés, cela contribue à reconfigurer dramatiquement la notion d'autorité. Celle-ci ne repose plus seulement dans Celui qui, du Haut, valide l'existence sociale des sujets en formation au-dessous de Lui. Elle relève bien plutôt d'une circulation de validité et de recevabilité, ou encore d'une autorisation circulaire qui se déroule bien plus horizontalement que verticalement, entre des esprits qui valent autant par leur masse que par leur statut plus ou moins privilégié. Dans la sphère des esprits, le Pouvoir se présente moins comme un pouvoir-agir que comme un pouvoir-d'être-cru. C'est ce que met assez suggestivement en scène un double épisode qui fait le corps de la troisième partie de Lamekis. 
Au sein d'un niveau narratif consacré aux aventures souterraines de Motacoa (le père adoptif de Lamekis), une série de monstres bizarres et hybrides attaquent le héros ainsi que son fidèle chien Falbao. Alors que la force physique supérieure de Falbao suffit à défaire les hommes-vers, quelque chose d' "extraordinaire» se passe au cours de l'affrontement avec les hommes-crapauds : au lieu que les combats se jouent à coups de poings, d'épées ou de morsures, c'est soudainement "un charme inconnu» qui paralyse l'invincible chien dès lors qu'un simple «étendard de la Chouette» captive ses regards : "ce vil aspect lui paroissoit redoutable, intimidoit ses regards", au point de neutraliser complètement sa force de combat et de résistance. Encerclé d'ennemis et privé de son plus vaillant adjuvant, Motacoa se croit perdu lorsqu'il s'aperçoit être lui-même porteur d'un «heureux ascendant»: «la fureur qui parut dans mes yeux, ou pour mieux dire, leur charme secret, causa un effroi si subit $\&$ si prodigieux à cette foule acharnée à ma perte qu' elle disparut une seconde fois en jetant des hurlemens affreux» (III, I5-I9). Les mêmes prodiges se reproduisant à plusieurs reprises, le héros en déduit que les aspects ont une puissance propre sur les individus - un charme - de par les impressions qu'ils font sur leur esprit, et il comprend du même coup que c'est moins par sa vigueur physique que par un enchantement similaire que Falbao était parvenu à défaire ses ennemis précédents (les Tumpingands) :

En examinant les choses avec précision, je ne pus m’empêcher de croire que je portois dans mes yeux l'assurance de ma victoire, \& que leur aspect donnoit des coups assurés à l'ennemi qui fuyoit. [...] L'aspect de cet hideux Oiseau étoit pour [Falbao] ce que le sien avoit été aux Tumpingands, \& je n'eus pas lieu bientôt de douter que le mien ne portât sur nos ennemis présens toute la force de ses meurtiers ascendans. [...] Sans des exemples journaliers de l'effet de ces antipathies, ne donneroit-on pas à ces vérités le nom de fictions? (III, I9, 23 \& 25)

On a donc ici l'exemple d'une fiction qui rencontre la réalité en y produisant des effets bien réels. Que la simple vue d'une Chouette, réellement inoffensive, puisse réellement terrasser un molosse cent fois plus fort qu'elle, voilà non seulement qui paraît relever de la fiction, mais voilà aussi qui souligne la puissance de la fiction, dès lors qu'il suffit de peindre une chouette sur un étendard (ou de feindre un regard furieux) pour obtenir les mêmes effets dans la réalité.

À travers cette scène de combat «symbolique», Motacoa découvre simultanément trois choses : $\mathrm{I}^{\circ}$ le simple aspect d'un être (l'apparence qu'il projette) contient une force propre d'impression (immatérielle) qui n'est nullement réductible à sa force physique (matérielle), selon l'exemple légendaire de la Méduse ou du serpent Basilic invoqué à propos de Falbao; 
$2^{\circ}$ on peut détruire ou produire ces objets particuliers dont l'aspect exerce un charme, un ascendant prodigieux, sur nos ennemis ou sur nos alliés; $3^{\circ}$ l'effectivité de tels charmes est d'ordre relationnel, puisqu'elle ne réside pas tant dans leur force interne que dans un certain pathos (une certaine sensibilité et réceptivité) propre à ceux auxquels on a affaire, de telle sorte que l'efficience de ces antipathies (ou de ces sympathies) doit être localisée entre celui qui voit et ce qui est vu (plutôt que dans l'un ou l'autre de ces pôles).

Cent Tumpingands disposent de la force matérielle pour écraser un chien, quelle que soit sa bravoure; Falbao dispose de la force matérielle nécessaire à transformer en pâtée un ou deux hommes-crapauds porteurs de drapeau. On ne comprend ce qui décide de l'issue réelle de tels affrontements qu'en prenant en compte quelque chose d'autre que la force matérielle : un effet de l'aspect des choses parfaitement disproportionné avec leur constitution matérielle objective. En continuité avec les longs chapitres dédiés par le roman à l'île des Sylphides - lieu propre des intelligences et des esprits je dirai qu'il faut se placer sur le plan de l'esprit dès lors que l'effet de l'impression ne s'explique plus seulement par la quantité ou par la qualité matérielles de la pression exercée, mais par une certaine disproportion entre le mouvement reçu et la réaction causée - disproportion qui définit une réceptivité subjective particulière.

\section{Puissance de la multitude}

Malgré ses allures monstrueusement chaotiques, le roman de Mouhy enchaîne très logiquement sur cette découverte de la puissance des apparences deux épisodes consacrés aux formes les plus frappantes, ainsi que les plus importantes, de cette disproportion entre les causes matérielles et les effets spirituels : l'expérience amoureuse et les conflits politiques ${ }^{9}$. Après avoir découvert pour la première fois les impressions que fait l'aspect d'une

9. Cette dimension politique de Lamekis paraît constitutive de son décor égyptien, s'il faut en croire N. Ferrand qui souligne que, dans le roman du premier XvıII ${ }^{\mathrm{e}}$ siècle, «la matière égyptienne aura affaire avec le pouvoir et la politique, il y sera question d'intrigues de palais, d'usurpation, de princes spoliés». Il apparaît que Mouhy se distingue du modèle qu'il parodie en rendant conflictuels les rapports entre autorité religieuse et pouvoir politique, là où une certaine image de l'Égypte les peignait en coexistence harmonieuse ("De l'érudition au fantastique dans la série des fictions égyptiennes au XviII ${ }^{\mathrm{e}}$ siècle : la parodie de Séthos (I73I) par Lamekis (I735-1738)", dans S. Menant et D. Quéro (éd.), Parodie et série dans la littérature française du XVIII siècle, Paris, Presses Paris Sorbonne, 2004, p. 352 et suiv.). 
femme aimée, en l'occurrence la princesse Nasilaé, Motacoa écoute celle-ci raconter l'histoire de sa vie, dont l'événement principal tourne autour d'un effort politique de transformation législative.

Tout part d'un pathos éprouvé par Nasilaé : «une antipathie effroïable pour la pratique de deux Loix qui répugnoient entièrement à $[s]$ a façon de penser» (III, 39). Les lois en question lui interdisaient de voir son père et lui enjoignaient de choisir un mari parmi de «vils prétendants». La princesse réussit à faire que son père, le bon Indiagar, roi des Amphicléocles, circonscrive la première loi et lui accorde une entrevue secrète. Malheureusement la Grand'-Prêtresse, la fourbe Lea-Minska, a vent de cette transgression et, au nom de la défense des traditions reçues, exige que Nasilaé soit exécutée et son père détrôné en punition de leur crime. S'ensuit un long conflit politique entre, d'une part, la Grand'-Prêtresse qui se sert des superstitions pour manipuler le peuple et, d'autre part, un roi éclairé auquel «la raison a enseigné à distinguer dans le nombre des Loix imposées, celles qui sont émanées de la sagesse divine d'avec celles qui sont enfantées par la politique de ses ministres" (III, 55). On retrouve ici en apparence le grand récit stéréotypé des Lumières : raison critique contre illusions superstitieuses, réformisme progressiste contre traditionalisme passéiste, bon prince éclairé contre prêtres et ministres corrompus.

L'intérêt et l'originalité de l'épisode tiennent cependant au statut central que jouent les manipulations du regard dans la captation des croyances de la multitude. Toute cette description des conflits politiques est en effet fondée sur un double renversement. D'une part, ce qui règne chez les Amphicléocles, ce n'est ni un roi, ni une Grand'-Prêtresse, mais les opinions de la multitude. Au sommet de son intensité, le conflit entre les deux rivaux (représentants du pouvoir monarchique et du pouvoir théologique) se résout dans une scène qui tient déjà du sondage ou du référendum comme vote de confiance. Les deux prétendants au pouvoir sont mis sur une machine en forme de balance, et ce sont les inclinations des spectateurs qui feront pencher la victoire dans l'un ou l'autre camp :

La Grand'-Prêtresse avoit la supériorité du thrône parce qu'elle représentoit le Dieu des Amphicléocles; mais de la Grande Tribune, l'Indiagar se trouvoit son égal, \& le peuple seul pouvoit faire tomber la balance du côté que son inclination lui dictoit. (III, 88)

Un texte de loi lu ultérieurement pour vider la querelle précisera lui aussi qu'il appartient "au peuple assemblé de porter un jugement définitif» (III, IO2). Dès lors que la décision ultime, le «jugement définitif», vient du public (des spectateurs), et plus précisément de ses «inclinations», qui 
font tomber la balance de l'autorité du côté qui lui plaira, c'est au bon plaisir de la multitude qu'est suspendu le pouvoir politique.

Le récit nous fait aborder un imaginaire politique qui remonte à $\mathrm{La}$ Boétie ou à Marsile de Padoue, et qui - à travers Spinoza - situe la source du pouvoir politique dans la "puissance de la multitude» (multitudinis potentia), que les institutions étatiques aussi bien que religieuses ne font que capter, généralement pour la détourner au profit de leurs dirigeants. Au sein de cet imaginaire qui ne représente plus le pouvoir comme venant du Haut pour s'abattre sur le peuple, mais qui le fait émaner d'une puissance à situer au sein de la basse multitude elle-même, l'épisode du conflit entre Indiagar et la Grand'-Prêtresse illustre de façon proprement exemplaire les stratégies de captation et de gestion des affects communs, des flux de croyances et de désirs qui constituent la substance même de la politique du point de vue de cette tradition philosophique.

Face à une opposante qui est parvenue à susciter un mouvement d'indignation au sein du peuple, le roi ne peut d'abord que "céder à la rumeur publique» (III, 56). Même lorsqu'il cherche à être un prince éclairé — et éclairant : un Enlightener, un Aufklärer - il ne doit jamais oublier que, contrairement à un "sujet ordinaire», qui peut se contenter de "jouir de lui-même» et de se « laisser conduire par la raison", le détenteur du pouvoir politique est condamné à être "l'esclave des apparences», des "préjugés», voire des «frénésies» de la multitude :

Cent fois, dans le dessein d'ouvrir les yeux à un peuple aveugle, j'ai tâché d'appuyer de si saintes vues du crédit de ceux qui ont droit d'entraîner leur suffrage; mais tel est l'entêtement, ô Princesse, de ceux même qu'une éducation élevée doit éclairer, le préjugé domine, la faiblesse du vulgaire a consacré ces fastueux usages! en vain la raison veut-elle percer cette ignorante obscurité, il semble que l'on se plaise dans ces ténèbres \& qu'on rougiroit de voir dissiper des nuages dont l'orgueil, l'indolence \& la mollesse sont les principes. (III, 64)

Derrière les anciens topoi du mépris condescendant des «élites» éclairées envers l'indécrottable ignorance des "masses», cet épisode de Lamekis fait apparaître à la fois une très intéressante acceptation de la force effective des croyances populaires et une suggestive attitude de stratégisation possible des facteurs qui gouvernent les flux de croyances et de désirs. Derrière l'opposition binaire (et réactionnaire) entre les lumières de la raison et «l'ignorante obscurité» où se complaît la «faiblesse du vulgaire» (orgueilleux, indolent et mou), Mouhy agence un discret retournement : c'est chez « ceux même qu'une éducation élevée doit éclairer» que "le préjugé domine». Plus subtilement (et plus radicalement), les flux de croyances sont décrits comme ayant plus de force que les actes de souveraineté : il ne s'agit pas 
d'assujettir ceux qui pensent mal aux ordres de ceux qui ont l'autorité de leur commander, mais seulement d'appuyer une proposition de réforme "sur le crédit de ceux qui ont droit d'entraîner leur suffrage». La formule est frappante : le «droit» ne vaut pas comme une source de pouvoir en soi (émanant d'une souveraineté supérieure), mais seulement comme ce qui contribue à asseoir et à augmenter le " crédit» (la crédibilité, la recevabilité) d'une action politique. On ne se situe pas ici dans un imaginaire de l'acte politique, mais dans un imaginaire de la circulation des flux de croyances, destinées à entraîner, selon leur intensité, plus ou moins de suffrages.

Conformément à l'allégorie sylphique, il n'est pas indifférent que cet imaginaire convoque des métaphores aériennes et vaporeuses pour s'inventer une expression digne de lui. Le roi, qui vient d'expliquer à sa fille que la raison était incapable de "dissiper les nuages» des illusions populaires, se dit confiant, quelques pages plus loin, que « sa seule présence alloit dissiper les nuages de la rébellion» (III, 70). C’est en effet l'air qui, depuis l'épicurisme antique, est perçu comme l'élément à travers lequel agissent des formes d'êtres très subtils, auxquels Lucrèce réservait le nom de simulacres. Comme dans les souterrains où se battaient Motacoa et son chien Falbao, c'est à travers une guerre des images que se résout le conflit politique entre Indiagar et la Grand'-Prêtresse. En plus de mettre en scène l'imaginaire de la démocratie radicale (fondée sur la potentia multitudinis), cet épisode donne aussi une leçon de stratégie politique, au terme de laquelle la captation des affects repose sur des propriétés très particulières de l'utilisation du spectacle.

\section{Puissance du simulacre}

Au sein de la machine à spectacle qui tranchera entre les deux prétendants au pouvoir politique, selon les inclinations et les suffrages de la multitude, c'est l'apparition de la figure du roi qui constitue le facteur de basculement du conflit, en suscitant «une acclamation générale : la majesté du Roi déracina dans un instant la rébellion $\&$ grava dans les cœurs les sentiments de respect \& d'amour» (III, 89). À l'inverse, c'est parce que la Grand'-Prêtresse ne peut pas se présenter aux regards à l'instant décisif que sa cause est perdue : «il fut heureux que la Loi qui défendoit au peuple de jetter les yeux sur elle eût lieu, cet aspect respectable étoit capable de le toucher $\&$ de le porter une seconde fois à la révolte» (III, IOS).

Comme dans les combats souterrains de Motacoa, c'est le pouvoir propre de l'aspect qui est le nerf de la guerre en politique. On croit ce qu'on voit 
- même si ce qu'on voit ne relève que d'un spectacle d'apparences. Les images qui flottent à travers les airs nous touchent et gravent en nous certains sentiments (indignation, respect) : c'est de ces impressions que résulte le maintien en place d'un pouvoir établi ou la réussite d'une rébellion. En une paronomase significative, Mouhy souligne que c'est l'aspect qui "contient dans le respect» (III, 85). La chose est à entendre littéralement : ce sont les images (les "aspects») qui capturent, canalisent et orientent ( «contiennent») les flux moléculaires de croyances et de désirs («respect») qui circulent au sein d'une société et qui animent sa vie spirituelle collective.

Outre l'image corporelle du roi ou de la Grand'-Prêtresse, ce sont bien entendu d'autres aspects qui peuvent produire des effets apparemment aussi "magiques» (c'est-à-dire disproportionnés à leurs seules propriétés physiques). Â côté de l'impression paralysante que peut causer un étendard de hibou sur l'intrépide Falbao, la scénographie politique rapportée par Nasilaé évoque divers objets investis d'aussi "extraordinaires" pouvoirs, dont une babouche et un $\mathrm{Ki}$-argouh représentant "une effigie du Roi». Une note explicative précise la nature de ces pouvoirs sur les «sujets» auxquels il est présenté : "Ils avoient une telle vénération pour l'aspect de ce signe, que lorsqu'il leur étoit montré, ils fermoient les yeux, comme se reconnaissant indignes de voir en face cette Effigie sacrée.» (III, 78)

On mesure le paradoxe visuel dont se nourrit ce type d'objet, pour lequel le récit réservera le terme de simulacre : on ne peut le voir qu'en fermant les yeux, et pourtant il faut l'avoir vu pour savoir/sentir qu'il faut fermer les yeux devant l'interdit de regard dont il est porteur. Le simulacre illustre le pouvoir propre d'une image qui simpose avec la plus grande force dont puisse disposer une image en même temps qu'elle s'abolit comme image. Si l'on essaie de reconstituer la hiérarchie des puissances qui structure le monde dépeint au sein de Lamekis, on s'aperçoit que ce ne sont pas les rois qui dominent les populations, mais les simulacres. Une autre note érudite précise en effet que «le Roi paroissait devant le Simulacre avec les mêmes cérémonies que ses Sujets observoient devant lui» (III, 90). Les princes de ce monde sont soumis au pouvoir des simulacres, de la même façon que les sujets sont soumis aux princes.

Une autre figuration suggestive du simulacre est proposée dans le même épisode à travers l'image du plus sacré ouvrage des Amphicléocles, le Livre d'airain. Comme de juste, il représente lui aussi un défi au regard : «lorsqu'on ouvroit le Livre des Loix, le privilège des yeux cessoit $\&$ le peuple étoit obligé de lui tourner le dos comme au Simulacre» (III, 96). Le Kafka de Vor dem Gesetz n'a pas rêvé pire : le livre des Lois ne peut s'ouvrir qu'avec les yeux fermés. Or les rares privilégiés qui ont l'autorisation de lire ce Livre d'airain y 
découvrent un «texte» des plus déroutants : «il n'étoit rempli que de points et de virgules, \& c'étoit la manière dont ces caractères étoient disposés qui signifioit les mots» (III, 80). Si l'on se souvient que les marques de ponctuation ont pour fonction de scander les flux de paroles, en indiquant les moments de pause et de respiration qu'il faut y insérer, on verra un autre paradoxe pragmatique dans la note érudite qui suit immédiatement cette description du Livre : «lorsque le Souverain des Amphicléocles permettoit à un de ses sujets de respirer, c'étoit lui commander de mourir» (III, 80)... Tuer quelqu'un en lui enjoignant de respirer est aussi difficile que lire un livre les yeux fermés. C'est dans le monde d'Orwell qu'on est cette fois plongé : le regard, c'est la cécité; la respiration, c'est la mort; le Souverain, c'est le peuple; la paix, c'est la guerre.

On est bien ici au cour des antipathies qui paraissent relever de la plus extraordinaire fiction, mais que le narrateur nous invite à ramener à des «exemples quotidiens». Or une autre note savante nous apprend que les Amphicléocles disposaient d'une procédure pour ne pas succomber à de telles antipathies - procédure qui s'inscrit dans l'imaginaire sylphique des «esprits élémentaires», que l'on peut contraindre à «rentrer dans leur tourbillon par la force de la grande Prière» :

Ces Peuples étoient persuadés que les atomes qu'ils respiroient étoient autant d'esprits purs ou impurs selon le bien ou le mal qu'ils faisoient; il étoit encore de foi parmi eux que lorsque la quantité des impurs s'étoient emparée d'eux \& en avoit chassé les bons esprits, ils mouroient subitement, \& qu'ils étoient transformés en reptiles affreux et toujours malheureux. Leur Théologie leur apprenoit à se garantir contre cette horrible infortune en prononçant trois mots mystérieux, qu'ils appeloient par excellence la grande Prière. (III, 50)

Le jeu des apparences et des croyances qui en résultent nous situe bien dans le monde des «esprits». Les simulacres et les aspects qui forcent le respect des membres d'une collectivité ne sont que des émanations subtiles et aériennes : des vapeurs, des esprits animaux — des influx neuronaux, dirait-on aujourd'hui en se croyant plus avancé. Le Livre d'airain n'est fait que du souffle des marques de ponctuation. C'est en nous forçant à respirer que le pouvoir souverain nous menace de mort et nous assujettit. Qu'est-ce donc que la grande Prière, sinon le souffle de trois mots qui, en sortant de notre bouche, repousse les mauvais esprits d'autres mots néfastes ou malintentionnés? Dans tous les cas, le merveilleux mis en scène dans Lamekis nous aide à percevoir les flux atomiques et moléculaires qui nous pénètrent, nous influencent, nous traversent et nous agissent, qu'ils s'insinuent en nous à travers la scansion de nos paupières ou qu'ils circulent en un va-et-vient rythmé par notre respiration. 


\section{La dynamique des faitiches}

J'espère avoir fait sentir à quel point la «monstruosité» apparente du roman de Mouhy recèle une force de suggestion et une densité signifiante bien propres à nous couper le souffle. Lépisode des Amphicléocles raconté par Nasilaé conduit Mouhy à engager son récit dans un emballement vertigineux. L'appareil des notes érudites permet à une imagination admirablement retorse de défier l'intelligence du lecteur en même temps qu'il donne à l'auteur l'occasion de saper joyeusement toute prétention d'autorité et de véridiction. Ces notes attribuées à Scaliger, Strabon, M. de Thou ou Mme Dacier — voire à un texte inédit d'Aristote lui-même - tendent tout à la fois à incarner et à déjouer par avance la critique ethnologique qui se met en place à l'époque du côté des récits de voyageurs. En dénonçant les "superstitions» des Amphicléocles à partir d'une posture d'iconoclaste, l'éditeur adopte le discours du Moderne qui, du haut de sa certitude rationaliste triomphante, dénonce l'inanité des croyances primitives dont s'illusionnent les autres cultures.

Pour conclure cette réflexion sur la croyance dans Lamekis, il convient de situer ce roman dans un triple contexte historique, qui l'investit d'enjeux essentiels au repérage de la mise en scène des «esprits» dans la littérature et l'imaginaire du XVIII ${ }^{\mathrm{e}}$ siècle. Le premier élément de contexte est justement fourni par la constitution du regard ethnologique qu'adoptent les Européens en rédigeant leurs récits de voyages (réels ou imaginaires) au contact d'autres cultures $^{10}$. Or il se trouve qu'un des textes importants de cette littérature, quelque peu postérieur à Lamekis, va inventer un mot qui s'efforce de désigner précisément le type d'efficience symbolique que Mouhy visait à travers sa mise en scène des simulacres. Et il se trouve que c'est également en référence (à peine moins fantaisiste) à l'Égypte que se fera cet épinglage ethnologique du simulacre.

Dans son ouvrage intitulé $D u$ culte des dieux fétiches ou Parallèle de l'ancienne religion de l'Égypte avec la religion actuelle de Nigritie, le président Charles de Brosses crée le néologisme "fétiche», tiré du portugais "Fetisso», qu'il traduit par "chose fée, enchantée, divine ou rendant des oracles» et qu'il fait remonter (de façon contestée aujourd'hui) à «la racine latine Fatum,

Io. Sur les résonances entre l'imaginaire ethnographique et la littérature merveilleuse, voir les beaux livres de L. Daston and K. Park, Wonders and the Order of Nature: II50-I750, New York, Zone Books, 200 I et de M. Baine Campbell, Wonder \& Science. Imagining Worlds in Early Modern Europe, Ithaca, Cornell University Press, 1999. 
Fanum, Fari ${ }^{\mathrm{II}}$ » : "ces Fétiches divins ne sont autre chose que le premier objet matériel qu'il plait à chaque nation ou à chaque particulier de choisir et de faire consacrer en cérémonie par ses Prêtres", ce sont "des choses douées d'une vertu divine, des oracles, des amulettes, \& des talismans", que «les Nègres», à la suite des Égyptiens, regardent «en général comme tutélaires pour les hommes $\&$ comme de puissans préservatifs contre toute sorte d'accidens ${ }^{12}{ }^{\prime \prime}$.

L'avertissement à la troisième partie de Lamekis avait déjà parfaitement identifié les fonctions du "fétiche» que le président de Brosses nommera vingt-cinq ans plus tard :

Je crois devoir encore assurer que cet Ouvrage porte avec lui le sacré talisman de la cabale la plus mystérieuse. Si mes lecteurs veulent bien s'en rapporter à ma parole, ils ne sortiront jamais sans ce livre admirable; il préserve de tous les maux, procure les aventures fortunées, éloigne les événemens bizarres \& capricieux, donne de l'esprit à ceux qui n'en ont pas. (III, v)

À première vue, l'auto-dérision dont fait preuve Mouhy paraît l'aligner sur le rejet drastique opéré par le président de Brosses quant à toute réalité effective des fétiches. Ce qui sera attribué par l'ethnologue à la «stupidité brute» des Nègres, le romancier en joue comme d'une crédulité incroyable, que l'on peut pousser à ses extrémités pour en faire un objet de persiflage - promettant aux lectrices que "tous leurs désirs seront accomplis avant la fin de l'année», pour autant qu'elles lisent le livre "le neuvième jour de mai à trois heures après minuit» (III, vii). Il coûte aussi peu de se moquer des femmes (lectrices de romans) que de mépriser les Nègres (fabricateurs de fétiches).

Un deuxième élément de contextualisation pourrait confirmer cette attitude commune, dès lors qu'on repère dans Lamekis des allusions discrètes mais suivies à l'affaire des Convulsionnaires. Il n'est guère difficile de reconnaitre les miracles à l'occasion desquels l'Esprit s'exprimait à travers les inspiré(e)s du cimetière de Saint-Médard, derrière la Grand'-Prêtresse qui fronde le pouvoir monarchique d'Indiagar en prophétisant la ruine du Royaume et en «se frappant la poitrine» "au pied du Simulacre» :

la force de son discours la conduisit bientôt dans l'enthousiasme divin des foudroyans oracles; tous ses traits changent, annoncent la convulsion; ses yeux sortent furieux de sa tête \& semblent verser des larmes de sang (III, 74).

II. C. de Brosses, Du culte des dieux fétiches ou Parallèle de l'ancienne religion de l'Égypte avec la religion actuelle de Nigritie, s. 1., I760, p. I8. Il s'était déjà servi de ce mot dans son Histoire des navigations aux terres australes de 1756.

I2. C. de Brosses, Du culte des dieux fétiches, ouvr. cité, p. II \& i8-i9. 
Ces scènes qui avaient défrayé la chronique des années I730, et qui continueront à hanter le siècle à travers les multiples « relations» de guérisons, de possessions et d'automutilations qui en seront faites jusqu'à la Révolution, sont clairement à situer à l'arrière-fond de l'imaginaire des "esprits». Elles se reconnaissent non seulement dans la confrontation politico-théologique entre le roi et la prêtresse, mais aussi en filigrane parodique des multiples scènes d'inspiration, d'initiation, d'épreuve et de supplice (de Dehahal) que multiplie Mouhy dans Lamekis.

De Brosses suggèrera d'ailleurs que les Convulsionnaires partageaient les illusions des fétichistes, lorsqu'il fera du «figurisme» la racine des superstitions qu'il dénonce ${ }^{13}$. Et c'est pour se moquer de tout figurisme que Mouhy - qui dédie des centaines de pages à peindre les merveilles de l'île des Sylphides, des esprits immatériels qui l'habitent et des intelligences supérieures qui nous en parviennent — s'amuse à rabaisser les souffles spirituels au statut malodorant de pet dans une note au récit hautement mystique de Dehahal : «un Auteur moderne a dit fort agréablement que lorsqu'un vent nous incommodoit, c'étoit un Sylphe que nous avions dans le corps, qui vouloit s'en échapper» (IV, Io6). C'est bien en iconoclaste radical que se comporte Mouhy dans ses notes, ses péritextes et ses mises en scène de la création littéraire, puisqu'il s'ingénie à casser toutes les images qu'il a produites lui-même et qui ne courent dès lors aucun risque de se transformer en idoles. Ne croire à rien — ou du moins à rien d'autre que les faits dûment établis par des procédures empiriques et rationnelles — voilà apparemment la position à laquelle nous convie le roman, comme tant d'autres textes célèbres des Lumières (encore en gestation en ces années 1730).

Malgré des analogies superficielles, la posture du romancier se distingue toutefois nettement de celle de l'ethnologue. En accomplissant le geste que Bruno Latour a identifié comme emblématique de l'attitude moderne, le second dénonce le fétichisme au nom d'un savoir qui vise à détruire la croyance (superstitieuse) pour lui substituer la raison (scientifique). Le geste accompli par Mouhy est beaucoup plus complexe et fondamentalement différent. On ne le comprend qu'en le resituant dans un troisième élément de contexte, fourni par le développement de genres littéraires centrés autour du merveilleux. Il est bien clair de Mouhy ne croit pas que son roman "porte avec lui le sacré talisman de la cabale la plus mystérieuse»,

13. Sur les Convulsionnaires et le figurisme dans les milieux jansénistes, voir les ouvrages de C. Maire, Les Convulsionnaires de Saint-Médard. Miracles, convulsions et prophéties à Paris au XVIII e siècle, Archives, Paris, Gallimard, 1985 ainsi que De la Cause de Dieu à la Cause de la Nation. Le Jansénisme au XVIII siècle, Bibliothèque des Histoires, Paris, Gallimard, I998, p. 237-365. 
qui assurera prospérité financière et sentimentale de son lecteur — pas plus que Perrault, Madame d'Aulnoy ou Hamilton ne «croyaient» à l'existence des fées ou que Galland ne croyait voir des génies sortir des bouteilles. Les conventions propres de la littérature merveilleuse tendent toutefois à déjouer simultanément les croyances et les dénonciations des croyances - lorsque ces dénonciations se font au nom d'une vérité ou d'une réalité dont l'existence serait, elle, bien établie.

Alors qu'en bon moderne, le président de Brosses dénonce les fétiches à partir d'une présomption de connaissance de certains faits bien établis, la fiction merveilleuse jongle avec des fées (et des sylphes) dont la dynamique tend à récuser la distinction même qu'établit le moderne entre faits et fétiches, entre objectivité et subjectivité, entre science et illusion, entre savoir et croyance. La sape de toute forme d'autorité dans les péritextes, le démembrement de toute figure d'autorialité dans les représentations de l'écriture, la mise en scène ridicule et outrée du geste même de dénonciation de la croyance superstitieuse au sein des notes faussement savantes — tout cela déstabilise aussi bien le statut des faits que celui des fétiches.

Mieux encore : deux siècles et demi avant que Bruno Latour n'en invente le mot, Mouhy (et avec lui toute une littérature merveilleuse de l'âge classique) met d'ores et déjà au jour la dynamique de ce mixte indissociable de fétiches et de faits qu'est le faitiche - à savoir une réalité qui est à la fois fabriquée par des pratiques humaines (comme le fétiche) et néanmoins dotée d'une existence autonome indépendante de nos désirs (comme le fait scientifique), tout en mettant en ouvre une efficience qui dépasse notre puissance d'agir individuelle (comme le fétiche encore). En analysant comment Pasteur découvre, isole et fait apparaître l'existence des microbes avec ses pratiques de laboratoire, ou comment la physique atomique découvre et génère une énergie nucléaire qui échappe à son contrôle de par l'intrication des pratiques de laboratoire dans le tissu des relations sociales et politiques, Bruno Latour nous invite à voir non seulement que les faits acquièrent leur autonomie parce quỉls sont fabriqués, mais aussi que les fabrications relèvent d'une efficience qui outrepasse toujours la maitrise de leurs fabricateurs. Prendre acte de ce que les faitiches sont la condition de notre puissance d'agir et d'argumenter implique de remettre en cause les dichotomies autour desquelles s'est constituée la modernité - entre l'objectif et le subjectif, la science et la croyance, la matière (extérieure) et l'esprit (intérieur). Cela conduit à mesurer à la fois notre capacité humaine à produire de l'être à travers nos fictions, et notre incapacité à contrôler $a$ priori ce que deviendront ces fabrications après leur réalisation collective au sein d'une nature dont nous ne sommes qu'une partie. D'où les vertus 
d'attention, de soin et de prudence (Care, Caute!) qu'exige le maniement des faitiches, dès lors qu'on reconnaît leur efficience de faitiches ${ }^{14}$.

\section{L'indépassable vérité du roman cabalistique}

C'est toute cette dynamique du faitiche qui s'esquisse à travers les lignes apparemment erratiques de Lamekis. Tout autant que par les effets "magiques" des aspects, des antipathies et des simulacres, Mouhy met en scène cette dynamique des faitiches principalement à travers ses références à la cabale. Sans aucunement "croire» que son livre ait les vertus magiques d'un "sacré talisman ", Mouhy décrit très précisément le fonctionnement de "cabales" qui ne manquent pas d'être bien «réelles», malgré tous les nuages d'illusion qui les entourent.

Dans l'épisode de la lutte entre le roi Indiagar et la Grand'-Prêtresse, le terme de "cabale» désigne la stratégie de spectacle politique à travers laquelle on tente de renverser un régime existant pour s'emparer du pouvoir. Cette stratégie consiste, on l'a vu, à savoir se servir des images (aspects, simulacres, fétiches) qui captent les désirs et les croyances de la multitude. Lorsqu'on peut dire d'un parti que «sa cabale, ses intrigues semblent triompher» (III, 56), on reconnaît qu'il a su jouer de fictions (fabriquées) pour s'emparer d'un certain pouvoir. C'est par le maniement efficace des faitiches que la cabale transforme quelque chose d'apparemment immatériel (un rêve, un espoir, une illusion, une vision, des paroles, les anglophones désigneraient ceci comme relevant du hot air) en un pouvoir devenu objectif, qui est en capacité de faire circuler des flots de richesses, d'emprisonner des corps, voire de couper des têtes.

En conformité avec une intuition qui traverse l'ensemble de Lamekis, le terme de "cabale» est toutefois plus précisément sollicité pour désigner une certaine machination de réceptivité. Lors du discours de réception de Dahahal parmi les Sylphes, on "entrevoit de la cabale» dans le fait que certains esprits chagrins "se récrient sur quelques épithètes" (IV, IOI) : on suspecte que leur écoute n'a pas été bienveillante, mais conditionnée par quelque jalousie ou conspiration sous-jacente. Dans l'avertissement de la cinquième partie, c'est l'auteur du roman qui se sait devoir faire face à «une cabale ignorante \& envieuse», prédisposée à le critiquer quoi qu’il écrive, et

14. B. Latour, Sur le culte moderne des dieux faitiches (1996), Paris, Les Empêcheurs de penser en rond, 2009 et L'Espoir de Pandore: Pour une version réaliste de l'activité scientifique (1999), Paris, La Découverte, 2007, chap. 8. 
contre laquelle il mobilise ses lecteurs, afin que ceux-ci publient ses louanges de façon préemptive $(\mathrm{V}, \mathrm{v})$. L'écoute d'un discours, la lecture d'un roman ne relèvent jamais d'une réception "ouverte» (vierge et inconditionnée) : elles sont toujours prédisposées par une certaine orientation préalable. Et c'est précisément autour de ces formes conditionnées de réceptivité que se jouent les luttes politiques et littéraires. Il ne suffit pas, pour un discours politique ou pour un roman, de simplement "dire» une vérité ou une fiction : ce qui importe le plus, c'est de composer une cabale qui s'insinue dans la réceptivité ambiante, pour la modifier de façon à neutraliser les cabales ennemies préexistantes.

C'est l'avertissement à la troisième partie de Lamekis qui déploie la complexité de la guerre des cabales dont dépend la réception d'un texte. L'auteur constate et déplore que «dans un nombre de gens qui nous font l'honneur de nous lire, il s'en trouve qui passent leur vie à faire des applications» (III, ii), c'est-à-dire à vouloir recevoir un récit purement fictionnel, fait pour "amuser " et "porter à la vertu», comme contenant des références et des attaques cryptées, visant telles personnes ou tels événements réels. Contre de tels soupçons, il déclare hautement «aimer mieux être loué par les endroits flatteurs de la simplicité que de [s]e faire un nom dans des cabales odieuses» (III, iii). Or c'est précisément dans ce troisième volume qu'il s'étend avec complaisance sur la description des cabales de la Grand'Prêtresse, dont les "convulsions" pouvaient difficilement ne pas évoquer, pour les contemporains, les débats générés autour des Jansénistes convulsionnaires, perçus comme défiant le pouvoir royal. Et c'est également dans ce même avertissement que l'auteur présentera son ouvrage, trois paragraphes plus loin, comme portant «avec lui le sacré talisman de la cabale la plus mystérieuse»...

On voit que Lamekis mérite pleinement d'être rangé au nombre des « romans cabalistiques». Le talisman propre à sa cabale est en effet exemplaire d'un dispositif poético-politique qui se situe au carrefour du regard ethnologique dénonciateur des fétiches, de la puissance des esprits mobilisée par l'épisode convulsionnaire et des propriétés du genre merveilleux. Loin de se réduire à un pur jeu de démolition (comme s'en contente la modernité iconoclaste envers les croyances "primitives"), la mise en scène des esprits élémentaires par le récit merveilleux dégage une positivité essentielle à la constitution du monde humain. La fable extraordinaire imaginée par Mouhy permet à la fois de nous faire prendre un certain recul par rapport aux rituels et aux simulacres qui conditionnent nos comportements sociaux (selon les virtualités du regard ethnologique), de nous faire mesurer la force spirituelle des courants collectifs de croyances et de désirs (qui agitent le corps des 
convulsionnaires) et de donner, à travers son roman merveilleux, l'exemple concret d'un simulacre qui se dénonce comme simulacre, sans pour autant perdre son efficience sur nos esprits. La poétique du merveilleux est en effet porteuse d'une leçon éminemment politique en produisant des faitiches qui, parce qu'ils se présentent explicitement comme des fétiches (comme des fictions fabriquées), nous permettent d'agir à la fois avec toute la puissance et avec toute la prudence dont relèvent les faitiches. 